Article

\title{
Nanostructure Formation on Diamond-Like Carbon Films Induced with Few-Cycle Laser Pulses at Low Fluence from a Ti:Sapphire Laser Oscillator
}

\author{
Seiya Nikaido, Takumi Natori, Ryo Saito and Godai Miyaji * (D) \\ Department of Applied Physics, Tokyo University of Agriculture and Technology, 2-24-16 Nakacho, \\ Koganei, Tokyo 184-8588, Japan; nikaidada573@gmail.com (S.N.); \\ impossible.is.nothing.1111@gmail.com (T.N.); s188097x@st.go.tuat.ac.jp (R.S.) \\ * Correspondence: gmiyaji@cc.tuat.ac.jp; Tel.: +81-42-388-7153
}

Received: 2 June 2018; Accepted: 14 July 2018; Published: 16 July 2018

\begin{abstract}
This study reports the results of experiments on periodic nanostructure formation on diamond-like carbon (DLC) films induced with $800 \mathrm{~nm}$, 7-femtosecond (fs) laser pulses at low fluence from a Ti:sapphire laser oscillator. It was demonstrated that 7-fs laser pulses with a high power density of $0.8-2 \mathrm{TW} / \mathrm{cm}^{2}$ at a low fluence of $5-12 \mathrm{~mJ} / \mathrm{cm}^{2}$ can form a periodic nanostructure with a period of $60-80 \mathrm{~nm}$ on DLC films. The period decreases with increasing fluence of the laser pulses. The experimental results and calculations for a model target show that 7-fs pulses can produce a thinner metal-like layer on the DLC film through a nonlinear optical absorption process compared with that produced with 100 -fs pulses, creating a finer nanostructure via plasmonic near-field ablation.
\end{abstract}

Keywords: femtosecond laser; laser ablation; nanostructure formation; surface plasmon polaritons; near-field; diamond-like carbon

\section{Introduction}

Superimposed femtosecond (fs) laser pulses can form a periodic nanostructure (PNS) on solid surfaces through ablation, where the period size $d$ is typically $10-20 \%$ of the laser wavelength $\lambda$ [1-6]. There has been considerable interest in this surface phenomenon for application in laser nanoprocessing, beyond the diffraction limit of light. Numerous studies have been conducted to understand the mechanism responsible for PNS formation [7-10]. The experimental conditions and laser parameters for PNS formation have been identified for various target materials, and the dominant physical mechanisms responsible for nanostructuring have been determined.

Based on a series of experiments and model calculations, Miyazaki and Miyaji found that PNS formation is induced by fs laser pulses at a moderate fluence $F$ through: a bonding structure change in the material [11-13]; generation of high-density electrons on the target surface, leading to the formation of a metal-like layer through linear and nonlinear optical absorption [13-15]; near-field ablation around the corrugated nanosurface [13-15]; and excitation of standing surface plasmon polariton (SPP) waves $[9,10,15-17]$. These laser-matter interaction processes can explain the origin and growth of PNSs on diamond-like carbon (DLC) [15], Si [16], GaN [10,17], Ti, and stainless steel [9], and theoretical calculations agree well with the observed nanoperiod, which is much smaller than $\lambda / 2$. Based on the physical mechanism, control methods for the PNS shape have been developed, allowing the formation of homogeneous nanogratings $[9,10,17]$ and a saw-like PNS [18] in air. However, some important processes for PNS formation are still unknown, and there is no consensus regarding the detailed mechanism.

For various kinds of material, it has been reported that the $d$ value for a PNS increases with increasing $F$ for the fs laser pulses at a fluence $F$ of a few $100 \mathrm{~mJ} / \mathrm{cm}^{2}$ to a few $\mathrm{J} / \mathrm{cm}^{2}$ with a power 
density $I$ of a few TW $/ \mathrm{cm}^{2}[7,8]$. Previous studies have concluded that this increase is attributed to the increasing thickness of the metal-like layer produced on the target material with increasing $F[16,17]$. However, this has never been experimentally confirmed.

The proposed mechanism of PNS formation suggests that a thin metal-like layer can be produced by the fs pulses at low $F$ with $I \sim \mathrm{TW} / \mathrm{cm}^{2}$ via a nonlinear absorption process, allowing confirmation of the thickness effect for nanostructuring. In this paper, we report the experimental results of PNS formation on DLC films irradiated with $800 \mathrm{~nm}$, 7-fs laser pulses with a high power density $I$ of $0.8-2 \mathrm{TW} / \mathrm{cm}^{2}$ and a low fluence $F$ of $5-12 \mathrm{~mJ} / \mathrm{cm}^{2}$ delivered from a laser oscillator. The results indicate the formation of a PNS with a period of $d=60-80 \mathrm{~nm}$ that decreases with increasing $F$. Based on the experimental results and a model calculation, it is shown that the excitation of SPPs at the interface between the thin metal-like layer and the DLC is certainly responsible for the nanostructuring process, and that the decrease of $d$ is attributed to the decreasing wavelength of the SPPs with increasing $F$ through an increase of electron density in the thin metal-like layer.

\section{Experimental}

Figure 1 shows a schematic diagram of the optical configuration used in the ablation experiments. As fs laser pulses with a high power density $I$ at low fluence $F$ can produce a thin metal-like layer on a target surface, the output of a Ti:sapphire laser oscillator was used in the experiments. The pulse duration $\Delta \tau$ was $\sim 7 \mathrm{fs}$, the wavelength $\lambda$ was $680-940 \mathrm{~nm}$, the repetition rate $f_{\text {rep }}$ was $80 \mathrm{MHz}$, and the pulse energy $U_{\text {pulse }}$ was $\sim 5 \mathrm{~nJ}$. The pulses were so-called few-cycle laser pulses, where the electromagnetic field oscillates for a few cycles [19]. The temporal and spectral profiles of the fs pulses were monitored with a spectral phase interferometry for direct electric-field reconstruction (SPIDER) device and a spectrometer, respectively. When measuring the temporal profile, a silver mirror was inserted to propagate the pulses to the SPIDER device. The output just after the oscillator had a negative group delay dispersion, which was compensated for to minimize the pulse duration by passing the beam through a beam splitter (thickness: $1 \mathrm{~mm}$ ) and a glass plate (thickness: $1 \mathrm{~mm}$ ). The laser pulses were spatially expanded with a pair of convex and concave silver mirrors and focused onto the target surface with a $\times 40$ Schwarzschild-type reflective objective (numerical aperture: 0.50 ) to a spot size $w_{0}$ of $\sim 2 \mu \mathrm{m}$ (1/ $e^{2}$ radius) on the surface, since the group delay dispersion had to be suppressed to obtain laser pulses with a high power density. A CMOS camera was used to image the focused beam on the target surface. The pulse energy $U_{\text {pulse just after the objective was measured with }}$ a pyroelectric detector, and the peak fluence $F=2 U_{\text {pulse }} /\left(\pi w_{0}^{2}\right)$ and the peak power density $I=F / \Delta \tau$ of the fs laser pulses on the target surface were estimated.

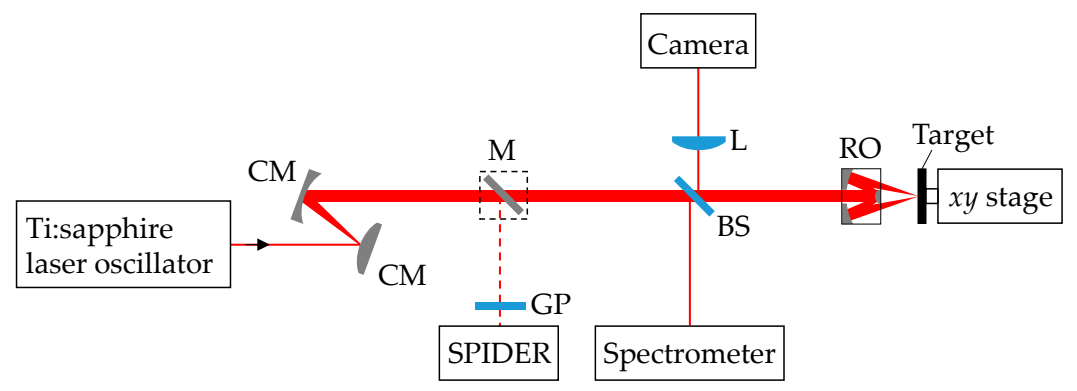

Figure 1. Schematic diagram of optical configuration for nanostructure formation. $\mathrm{CM}$, convex or concave mirror; $\mathrm{M}$, mirror; GP, glass plate; BS, beam splitter; L, lens; RO, reflective objective.

As the target, we used a DLC film (thickness: $1.7 \mu \mathrm{m}$ ) that was deposited on a polished silicon substrate with a plasma-based ion implantation system. The root-mean-square value of surface roughness was measured to be less than $1 \mathrm{~nm}$ with a scanning probe microscope (SPM). The target was set on an $x y$ motorized stage, which could move at a constant speed $v$ of $0.1-100 \mu \mathrm{m} / \mathrm{s}$. The surface morphology was observed using a scanning electron microscope (SEM) and the SPM. 
A two-dimensional Fourier transform was applied to the SPM images to analyze the distribution of the spatial periodicity in the surface structure along the polarization direction. The bonding structure of the target surface irradiated with the fs pulses was analyzed using micro-Raman spectroscopy with a diode-pumped, single-longitudinal-mode, $532 \mathrm{~nm}$ laser beam focused with a $\times 40$ objective.

\section{Results and Discussion}

Figure 2a-c show SEM and SPM images and spatial frequency spectra of DLC films irradiated with $7 \mathrm{fs}$ pulses with $I=1 \mathrm{TW} / \mathrm{cm}^{2}$ at $F=6 \mathrm{~mJ} / \mathrm{cm}^{2}$ for $v=0.1-10 \mu \mathrm{m} / \mathrm{s}$. For $v=100 \mu \mathrm{m} / \mathrm{s}$, the surface was observed to swell and was not ablated because of the small shot number of the laser pulses onto the target surface. When $v$ was decreased to $10 \mu \mathrm{m} / \mathrm{s}$ (i.e., the shot number increased), the formation of a PNS with a period $d$ of $\sim 50 \mathrm{~nm}$ was observed on the ablated DLC surface, as shown in Figure $2 \mathrm{a}$. The line-like structure was perpendicular to the direction of polarization. When $v$ was decreased to $1 \mu \mathrm{m} / \mathrm{s}$, a PNS with $d$ of $\sim 70 \mathrm{~nm}$ formed, as shown in Figure $2 \mathrm{~b}$. With a further decrease of $v$ to $0.1 \mu \mathrm{m} / \mathrm{s}$, deeper ablation traces with $d$ of $\sim 80 \mathrm{~nm}$ formed, as shown in Figure 2c. For comparison, the target surfaces were also irradiated by 100 -fs laser pulses with $I=0.1 \mathrm{TW} / \mathrm{cm}^{2}$ at the same $F$. These pulses were produced by a glass plate (thickness: $3 \mathrm{~mm}$ ) positioned just after the laser oscillator. As shown in Figure 2d, a PNS did not form on the ablated surface under these conditions.

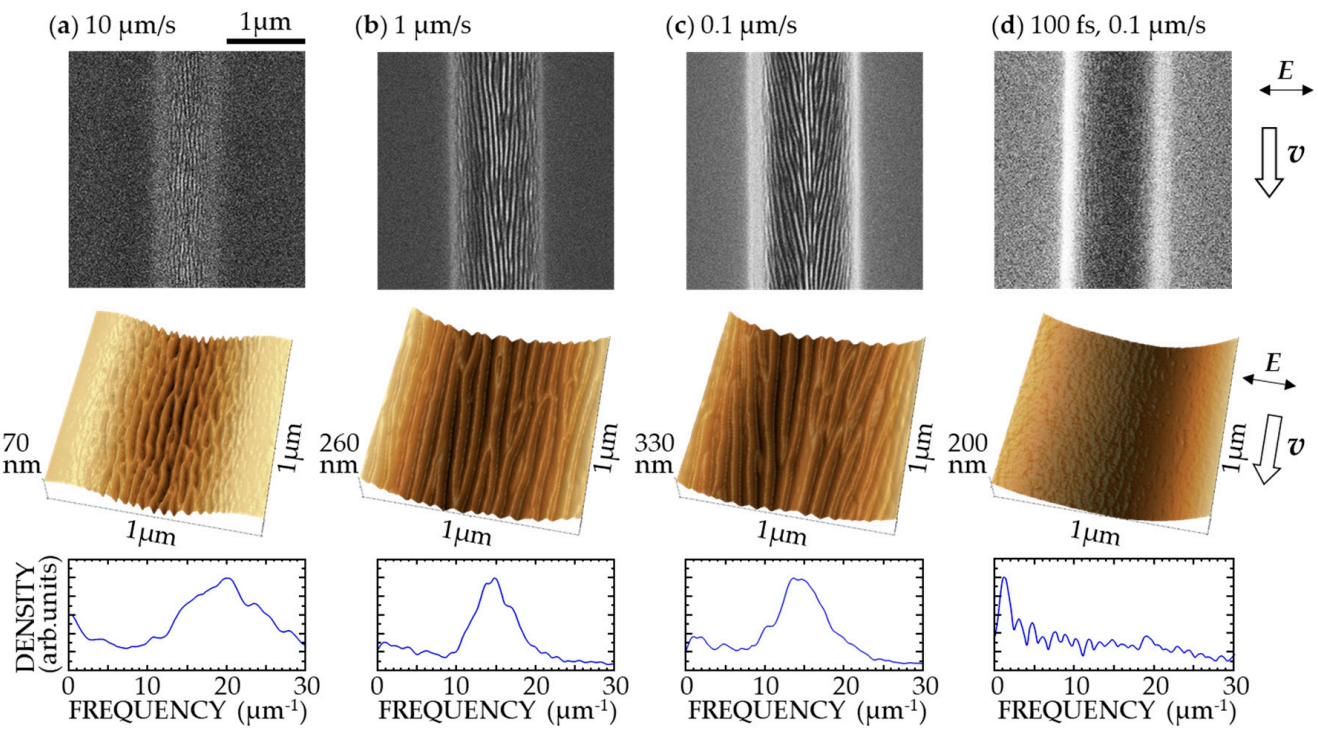

Figure 2. Scanning electron microscopy (SEM) images (top), scanning probe microscopy (SPM) images (middle), and spatial frequency spectra (bottom) of a diamond-like carbon (DLC) film surface irradiated with 7-fs pulses, with $I=1 \mathrm{TW} / \mathrm{cm}^{2}$ at $F=6 \mathrm{~mJ} / \mathrm{cm}^{2}$ for $(\mathbf{a}) v=10 \mu \mathrm{m} / \mathrm{s},(\mathbf{b}) v=1 \mu \mathrm{m} / \mathrm{s}$, and (c) $v=0.1 \mu \mathrm{m} / \mathrm{s}$, and (d) those irradiated with 100-fs pulses with $I=0.1 \mathrm{TW} / \mathrm{cm}^{2}$ at $F=6 \mathrm{~mJ} / \mathrm{cm}^{2}$ for $v=0.1 \mu \mathrm{m} / \mathrm{s}$. $E$ and $v$ denote directions of polarization and laser scanning, respectively.

In previously reported experiments, PNSs formed on DLC films with 100-fs laser pulses with $I=1-2 \mathrm{TW} / \mathrm{cm}^{2}$ at $F=100-200 \mathrm{~mJ} / \mathrm{cm}^{2}$, delivered from a chirp-pulse amplification Ti:sapphire laser system [3,11-15]. The results shown in Figure 2 suggest possible laser-matter interaction processes for PNS formation, as discussed in previous studies [13-16]. As $v$ is decreased, a bonding structure change-from DLC to glassy carbon (GC) — is induced in the surface layer. This produces nanometer surface roughness due to swelling of the material, as a thin layer with a high electron density is produced on the surface through a nonlinear optical absorption process. On the highly curved swollen metal-like surface, an intense near-field is generated that enhances the incident electric field and initiates nanoscale ablation. Then, SPPs are transiently excited via coherent coupling of the incident laser pulses with the corrugated surface, where the GC layer, including high-density electrons, works as 
a thin metal layer between air and the DLC for the excitation of SPPs [20]. The periodic enhancement of the near-field of SPPs excited in the surface layer induces ablation, which forms a PNS on the surface. The experimental results shown in Figure 2 indicate that such a process occurs sufficiently when a DLC film is irradiated with 7-fs pulses with a high density of $1 \mathrm{TW} / \mathrm{cm}^{2}$ at a low fluence of $6 \mathrm{~mJ} / \mathrm{cm}^{2}$.

An increase in $F$ is expected to increase the density of the free electrons produced in the surface layer, leading to a change in surface morphology. To confirm this, surfaces were ablated with 7-fs pulses for $v=0.1 \mu \mathrm{m} / \mathrm{s}$ for $F=5-12 \mathrm{~mJ} / \mathrm{cm}^{2}$, corresponding to $I=0.8-2 \mathrm{TW} / \mathrm{cm}^{2}$. The results are shown in Figure 3. At the lowest $F$, multiple shots produced a PNS with $d \sim 85 \mathrm{~nm}$; at the highest $F$, multiple shots produced a finer PNS with $d \sim 60 \mathrm{~nm}$. Figure 4 plots the $d$ value obtained from the isolated peak position in the Fourier spectrum of the SPM images as a function of $F$ and $I$. With increasing $F, d$ decreases from about 85 to $60 \mathrm{~nm}$. For irradiation with 100-fs laser pulses with $I=1-4 \mathrm{TW} / \mathrm{cm}^{2}$ at $F=100-400 \mathrm{~mJ} / \mathrm{cm}^{2}$, it has been reported that the $d$ value of the PNSs formed on various kinds of material (e.g., DLC, TiN, stainless steel, $\mathrm{Ti}$, Si, and $\mathrm{GaN}$ ) increased with increasing $F[3,9,16,17]$, which is opposite to the results obtained in the present study. This suggests that low-fluence fs pulses with a high power density play a crucial role in the surface morphological change that leads to nanostructuring.

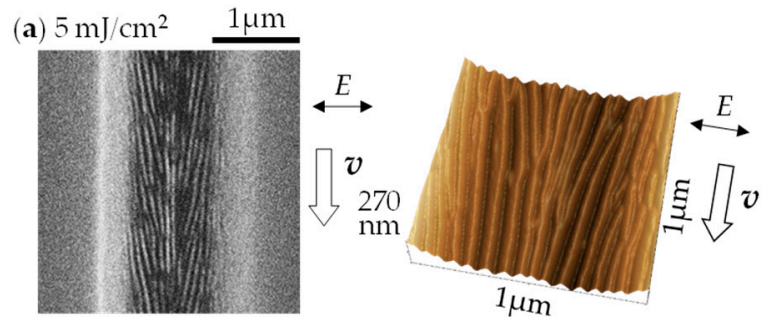

(b) $12 \mathrm{~mJ} / \mathrm{cm}^{2}$
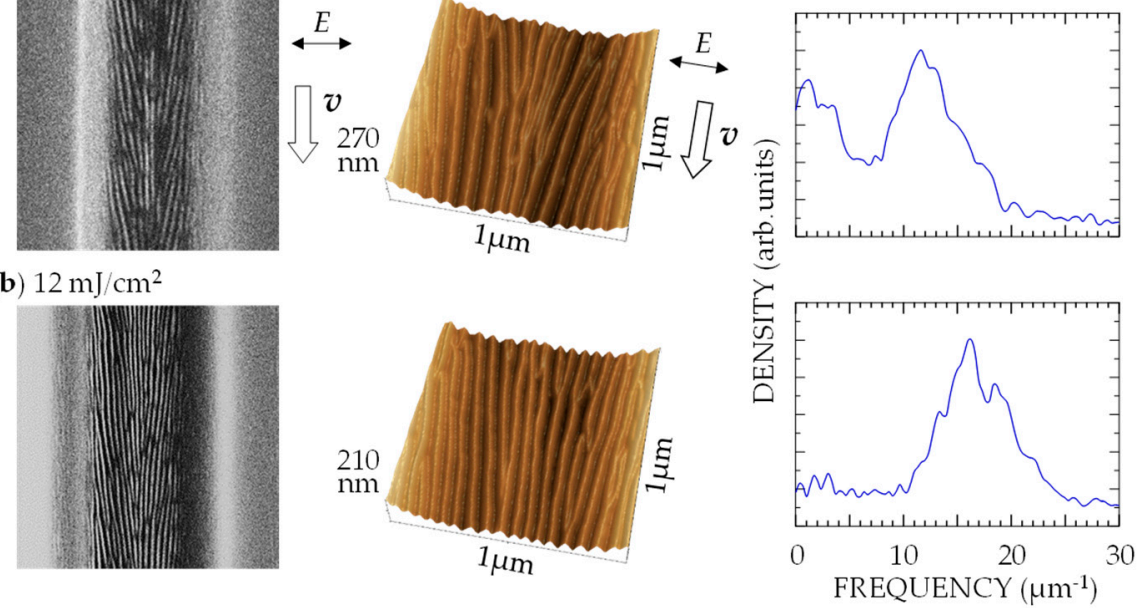

Figure 3. SEM images (left), SPM images (center), and spatial frequency spectra (right) of DLC film surface irradiated with 7-fs pulses at $v=0.1 \mu \mathrm{m} / \mathrm{s}$ for $(\mathbf{a}) I=0.8 \mathrm{TW} / \mathrm{cm}^{2}, F=5 \mathrm{~mJ} / \mathrm{cm}^{2}$ and $(\mathbf{b}) I=2 \mathrm{TW} / \mathrm{cm}^{2}$, $F=12 \mathrm{~mJ} / \mathrm{cm}^{2}$. $E$ and $v$ denote directions of polarization and laser scanning, respectively.

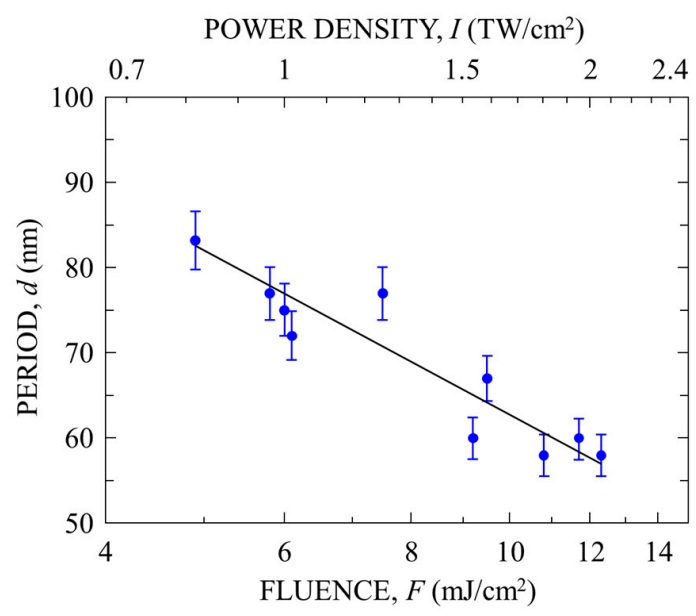

Figure 4. Period $d$ of a periodic nanostructure (PNS) on DLC film formed with 7-fs laser pulses as a function of $F$ and $I$ for $v=0.1 \mu \mathrm{m} / \mathrm{s}$. 
In a previous study, we reported that PNS formation on a DLC surface is preceded by a change in the bonding structure, from DLC to GC [13]. The swelling of the target surface observed for $v=100 \mu \mathrm{m} / \mathrm{s}$ indicates that the onset of ablation at $v \leq 10 \mu \mathrm{m} / \mathrm{s}$ is preceded by a change in the bonding structure to GC in the target surface. To confirm this, Raman spectra were obtained from surfaces ablated with 7-fs pulses with $I=1 \mathrm{TW} / \mathrm{cm}^{2}$ at $F=6 \mathrm{~mJ} / \mathrm{cm}^{2}$ for $v=0.1 \mu \mathrm{m} / \mathrm{s}$. The results are shown in Figure 5, together with spectra of surfaces ablated with 100 -fs pulses with $I=0.1 \mathrm{TW} / \mathrm{cm}^{2}$ at $F=6 \mathrm{~mJ} / \mathrm{cm}^{2}$ for $v=0.1 \mu \mathrm{m} / \mathrm{s}$ and non-irradiated DLC for comparison. Each spectrum is normalized to give a maximum intensity of unity. The asymmetric broad spectrum for the non-irradiated DLC has a single peak at $1530 \mathrm{~cm}^{-1}$, which mainly consists of two spectra at peaks at $\sim 1360 \mathrm{~cm}^{-1}$ (D band) and $\sim 1590 \mathrm{~cm}^{-1}$ (G band) [21]. The D and G bands are attributed to bond angle disorder in $s p^{2}$ graphite-like micro/nanodomains and bond stretching between pairs of $s p^{2}$ atoms in both the rings and chains, respectively. The ratio of the intensities of the $\mathrm{D}$ and $\mathrm{G}$ peaks $\left(I_{D} / I_{G}\right)$ and the position of the $\mathrm{G}$ peak have been reported to indirectly indicate the composition ratio of $s p^{2}$ and $s p^{3}$ bonding structures in DLC films [22-24]. These reports have shown that an increase in $I_{D} / I_{G}$ and a shift of the $G$ peak to a higher frequency represent an increase in the amount of $s p^{2}$ structures. The spectra from surfaces ablated with 7-fs and 100-fs pulses, shown in Figure 5, clearly show two spectral peaks at 1355 and $1590 \mathrm{~cm}^{-1}$, respectively, indicating an increase in disordered carbon or GC [25-28]. As shown in Figure $5 b, I_{D}$ for the surface irradiated with 7-fs pulses is smaller than that for the surface irradiated with 100-fs pulses. In addition, the position of the G peak for 7-fs pulses is shifted less than that for 100 -fs pulses. These results show that less GC existed in the target surface irradiated with 7-fs laser pulses compared to that which existed with 100-fs pulses, despite the same $F$.
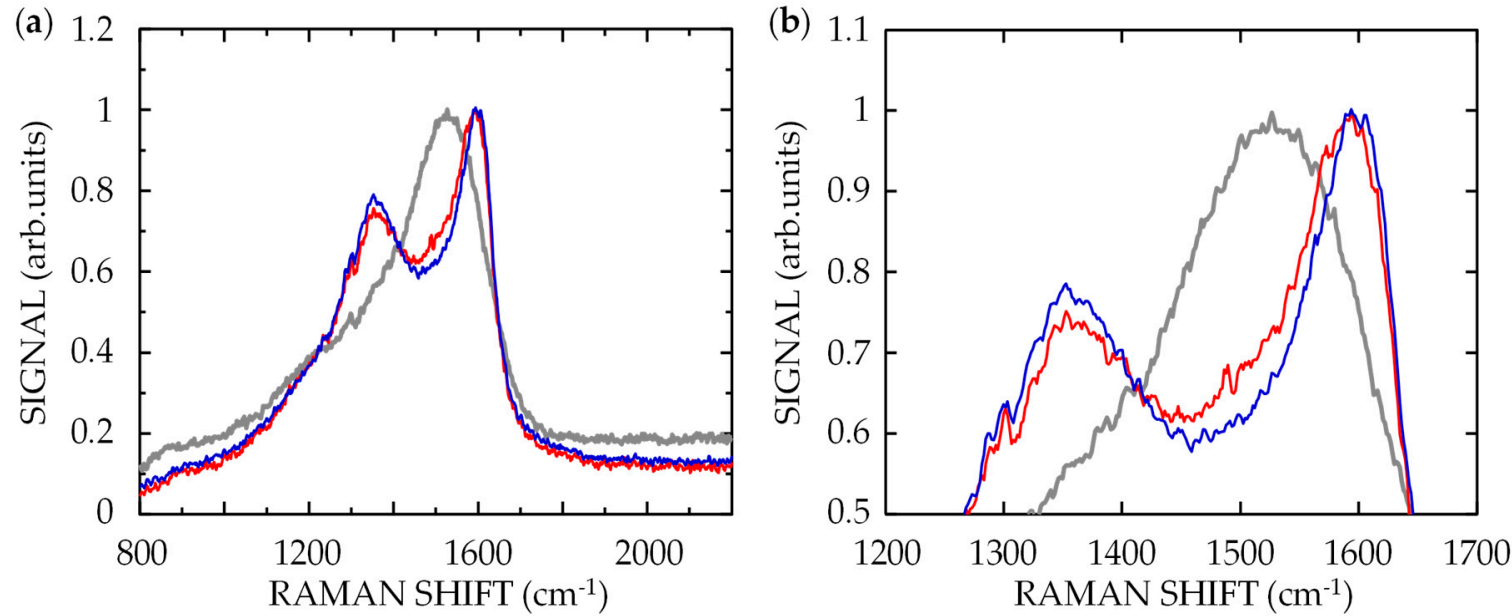

Figure 5. (a) Raman spectra of non-irradiated DLC film (gray) and DLC films irradiated with 7-fs (red) and 100-fs (blue) pulses at $F=6 \mathrm{~mJ} / \mathrm{cm}^{2}$ for $v=0.1 \mu \mathrm{m} / \mathrm{s}$; (b) expanded spectra of (a) in the vicinity of the peaks of $\mathrm{D}$ and $\mathrm{G}$ bands.

To examine the the bonding structural change and ablation processes in detail, Raman spectra were obtained from a DLC film irradiated with 7 fs pulses with $I=1 \mathrm{TW} / \mathrm{cm}^{2}$ at $F=6 \mathrm{~mJ} / \mathrm{cm}^{2}$ for various values of $v(v=0.1-100 \mu \mathrm{m} / \mathrm{s})$. For comparison, spectra were also obtained from a film irradiated with $100 \mathrm{fs}$ pulses with $I=0.1 \mathrm{TW} / \mathrm{cm}^{2}$ at $F=6 \mathrm{~mJ} / \mathrm{cm}^{2}$. The peak intensities and positions of the $D$ and $G$ bands in the spectra were identified using a curve-fitting program with the Lorentzian function [29]. Figure 6a shows $I_{D} / I_{G}$ plotted as a function of $v$. In the spectrum of the non-irradiated DLC film, $I_{D} / I_{G}$ was $\sim 1.25$. For $v=100 \mu \mathrm{m} / \mathrm{s}$, the ratio for both 7 -fs and 100-fs pulses increased to $\sim 1.5$. With a decrease in $v$, the ratio monotonically increased, with that for 7 -fs pulses being smaller than that for 100 -fs pulses. Figure $6 \mathrm{~b}$ shows the position of the $\mathrm{G}$ peak plotted as a function of $v$. In the spectrum of the non-irradiated DLC film, the G peak position was $1582 \mathrm{~cm}^{-1}$. For $v=100 \mu \mathrm{m} / \mathrm{s}$, the position for both 7-fs and 100-fs pulses shifted to $\sim 1590 \mathrm{~cm}^{-1}$. With decreasing $v$, the position monotonically 
shifted to higher frequencies, with that for 7-fs pulses being at lower frequencies than that for $100-\mathrm{fs}$ pulses. These results show two crucial processes for surface modification and subsequent ablation. For $v=100 \mu \mathrm{m} / \mathrm{s}$, where both 7-fs and 100-fs pulses with the same $F$ induced only swelling and no ablation on the target, the change in the spectra shown in Figure 6 indicates that the amount of GC at the surfaces irradiated with 7-fs and 100-fs pulses is the same, and that the surface phenomena do not depend on $I$. For $v \leq 10 \mu \mathrm{m} / \mathrm{s}$, where both 7 -fs and 100-fs pulses with the same $F$ induced not only a bonding structure change but also ablation on the target, the experimental results indicate that 7-fs pulses with higher $I$ were strongly absorbed near the target surface through a nonlinear optical absorption process, forming a thinner GC layer than that produced by $100-\mathrm{fs}$ pulses. The surface of the layer was then ablated.

(a)

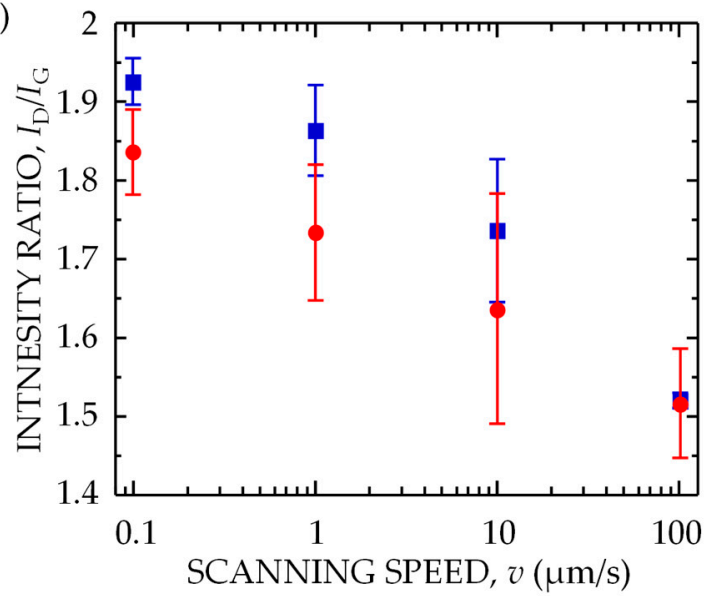

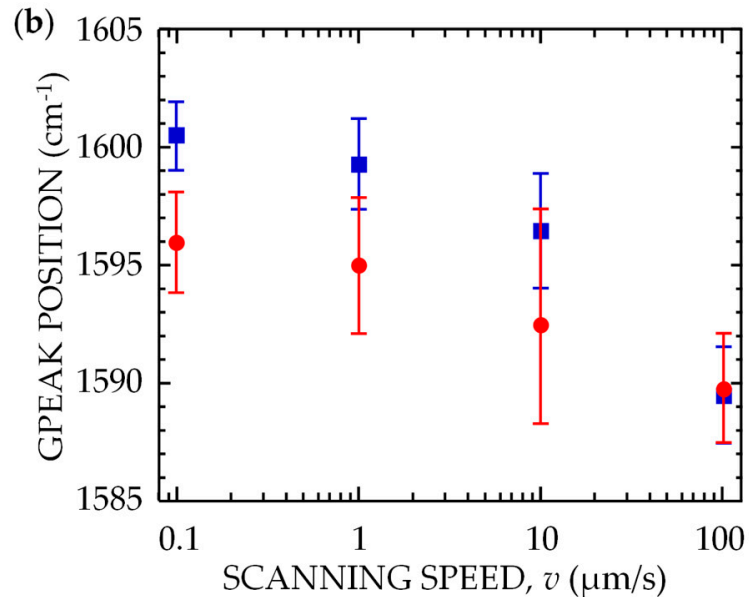

Figure 6. (a) Ratio of intensities of $D$ and $G$ peaks $\left(I_{D} / I_{G}\right)$ and (b) position of $G$ peak for DLC films irradiated with 7-fs laser pulses with $I=1 \mathrm{TW} / \mathrm{cm}^{2}$ at $F=6 \mathrm{~mJ} / \mathrm{cm}^{2}$ (red circles) and those irradiated with 100-fs laser pulses with $I=0.1 \mathrm{TW} / \mathrm{cm}^{2}$ at $F=6 \mathrm{~mJ} / \mathrm{cm}^{2}$ (blue squares) as function of scanning speed $v$.

Based on these experimental results and the physical mechanism for nanostructuring $[8,15,16,30]$, the origin of the decrease in $d$ with increasing $F$ is discussed. The SPP wavelength $\lambda_{\text {spp }}$ was calculated for the model surface illustrated in the inset of Figure 7, where it was assumed that the fs laser pulses are incident on the target in air, free electrons are produced at the GC surface to form a thin metal-like layer on the DLC substrate, and SPPs are excited at the interface between the metal-like layer and the DLC. The calculation method was almost the same as that used in our previous studies $[15,16]$. Briefly, $\lambda_{\mathrm{spp}}=2 \pi / \operatorname{Re}\left[k_{\mathrm{spp}}\right]$ was calculated using the following relation between light and SPPs:

$$
k_{\mathrm{spp}}=k_{0}\left[\varepsilon_{\mathrm{DLC}} \varepsilon^{*} /\left(\varepsilon_{\mathrm{DLC}}+\varepsilon^{*}\right)\right]^{1 / 2}
$$

where $k_{0}$ is the wavevector of the incident light in vacuum, and $\varepsilon^{*}$ and $\varepsilon_{\text {DLC }}$ are the relative dielectric constants for the metallic GC and the DLC, respectively. As the GC layer is ionized by fs laser pulses, $\varepsilon^{*}$ rapidly changes during the interaction as:

$$
\varepsilon^{*}=\varepsilon_{\mathrm{GC}}-\left[\omega_{\mathrm{p}}^{2} /\left(\omega^{2}+i \omega / \tau\right)\right]
$$

where $\varepsilon_{\mathrm{GC}}$ is the static dielectric constant for the GC layer, and the second term represents the effect of free electrons with a density of $N_{\mathrm{e}}$ produced in the GC layer, where $\omega$ is the laser frequency in vacuum, $\tau=1 \mathrm{fs}$ is the Drude damping time for free electrons [31,32], and $\omega_{\mathrm{p}}=\left[e^{2} N_{\mathrm{e}} /\left(\varepsilon_{0} m^{*} m\right)\right]^{1 / 2}$ is the plasma frequency, with the dielectric constant of vacuum $\varepsilon_{0}$, electron charge $e$, electron mass $m$, and optical effective mass of electrons $m^{*}=1$. In the calculation, because the wavelength of the 7 -fs laser pulse used in the present experiment was 680-940 nm, the static dielectric constants for DLC and GC were 
used for three wavelengths: $\varepsilon_{\mathrm{DLC}}=6.9+i 3.8$ and $\varepsilon_{\mathrm{GC}}=3.0+i 2.8$ for $\lambda=600 \mathrm{~nm} ; \varepsilon_{\mathrm{DLC}}=8.0+i 2.9$ and $\varepsilon_{\mathrm{GC}}=3.1+i 3.1$ for $\lambda=800 \mathrm{~nm}$; and $\varepsilon_{\mathrm{DLC}}=8.5+i 2.6$ and $\varepsilon_{\mathrm{GC}}=3.6+i 4.5$ for $\lambda=1000 \mathrm{~nm}$ [33].

Figure 7 shows the period of the PNS, $D=\lambda_{\text {spp }} / 2=\pi /\left(\operatorname{Re}\left[k_{\text {spp }}\right]\right)$, calculated for $\lambda=600,800$, and $1000 \mathrm{~nm}$ as a function of $N_{\mathrm{e}}$. The excitation of SPPs at the interface between the metallic GC layer and the DLC is allowed for $\operatorname{Re}\left[\varepsilon^{*}\right] \times \operatorname{Re}\left[\varepsilon_{\mathrm{DLC}}\right]<0$ [20], which corresponds to the regions of $N_{\mathrm{e}}>1.0 \times 10^{22} \mathrm{~cm}^{-3}$ for $\lambda=600 \mathrm{~nm}, N_{\mathrm{e}}>6.4 \times 10^{21} \mathrm{~cm}^{-3}$ for $\lambda=800 \mathrm{~nm}$, and $N_{\mathrm{e}}>5.2 \times 10^{21} \mathrm{~cm}^{-3}$ for $\lambda=1000 \mathrm{~nm}$. With increasing $N_{\mathrm{e}}, D$ decreases from $\sim 200 \mathrm{~nm}$ to $\sim 100 \mathrm{~nm}$. Because $N_{\mathrm{e}}$ should increase with increasing $I$ via stronger nonlinear optical absorption, the decrease in $D$ with increasing $N_{\mathrm{e}}$ is in good agreement with the decrease in $d$ with increasing I for 7-fs laser pulses shown in Figure 4.

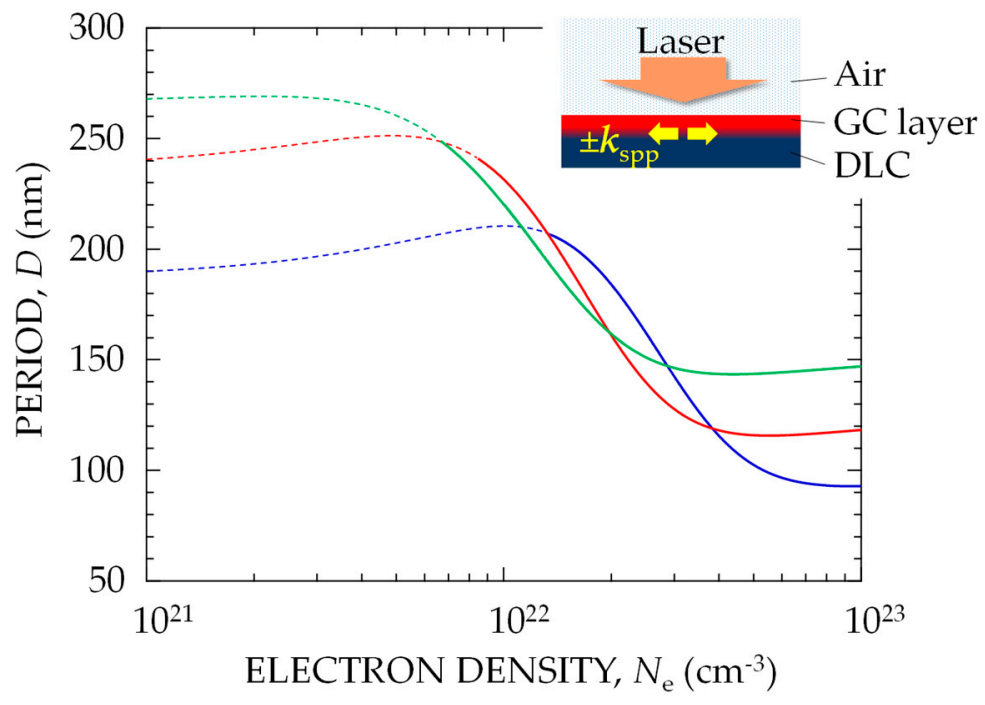

Figure 7. Calculated groove period $D$ as function of $N_{\mathrm{e}}$ in the glassy carbon (GC) layer at $\lambda=600 \mathrm{~nm}$ (blue), $800 \mathrm{~nm}$ (red), and $1000 \mathrm{~nm}$ (green), where the excitation of surface plasmon polaritons (SPPs) is allowed in the region (solid curves) of $0<\operatorname{Re}\left[\varepsilon^{*}\right]$. The inset shows a schematic drawing of the initial target surface modeled for calculation. SPPs (left/right arrows) are excited at the interface between the DLC and GC layers by a high density of electrons produced by irradiation using high power -density laser pulses (down arrow).

The present experimental and calculation results show that the period $d$ for a PNS was smaller than $D$, and that the $d$ value for a PNS formed with high-fluence 100-fs laser pulses was similar to $D$ for $\lambda=800 \mathrm{~nm}$, which is consistent with the results of a previous study [15]. Regarding the excitation of SPPs on a thin metal film, it has been reported that the wavenumber of the SPPs increases with decreasing thickness of the film because of an increase in the radiation damping of SPPs [20]. These results suggest that $d$ being smaller than $D$ can be attributed to the excitation of SPPs with a larger wavenumber by the thinner metallic layer produced with 7-fs laser pulses. A calculation model for $D$ that includes the effect of the metallic layer thickness will be presented and discussed in a separate paper. To discuss the formation process of PNS in detail and make a more accurate model for the nanostructuring, we need to quantitatively measure the amount and thickness of the GC layer on DLC film by using advanced techniques, such as a grazing-incidence small-angle X-ray scattering [34-36].

\section{Conclusions}

This study examined the PNS that formed on a DLC film with 7-fs laser pulses at a low fluence from a laser oscillator. The results show the formation of a PNS with a period of $d=60-80 \mathrm{~nm}$ and a decrease in $d$ with increasing fluence. Based on the experimental results and a model calculation, it is shown that the excitation of SPPs at the interface between the thin metal-like layer and the DLC is 
certainly responsible for the nanostructuring process, and that the decrease of $d$ is attributed to the wavelength of the SPPs decreasing with increasing $F$ due to an increase of electron density in the thin metal-like layer.

Author Contributions: S.N. and R.S. performed the ablation experiments; T.N. fabricated the micro Raman spectroscope and analyzed the bonding structure of ablated surfaces; G.M. designed the experiments and made the physical model; all the authors wrote this paper together.

Funding: This research was supported in part by the Amada Foundation 2015 and Joint Usage/Research Program on Zero-Emission Energy Research, Institute of Advanced Energy, Kyoto University (ZE29C-1, 2017).

Acknowledgments: The authors would like to thank K.S. for the preliminary experiments, K.M. for helpful comments and useful discussions on Raman spectroscopy, and A.H. and Y.H. for the composition analysis of DLC films.

Conflicts of Interest: The authors declare no conflict of interest.

\section{References and Note}

1. Bonse, J.; Sturm, H.; Schmidt, D.; Kautek, W. Chemical, morphological and accumulation phenomena in ultrashort-pulse laser ablation of TiN in air. Appl. Phys. A Mater. Sci. Process. 2000, 71, 657-665. [CrossRef]

2. Reif, J.; Costache, F.; Henyk, M.; Pandelov, S.V. Ripples revisited: Non-classical morphology at the bottom of femtosecond laser ablation craters in transparent dielectrics. Appl. Surf. Sci. 2002, 197-198, 891-895. [CrossRef]

3. Yasumaru, N.; Miyazaki, K.; Kiuchi, J. Femtosecond-laser-induced nanostructure formed on hard thin films of TiN and DLC. Appl. Phys. A Mater. Sci. Process. 2003, 76, 983-985. [CrossRef]

4. Wu, Q.; Ma, Y.; Fang, R.; Liao, Y.; Yu, Q. Femtosecond laser-induced periodic surface structure on diamond film. Appl. Phys. Lett. 2003, 82, 1703-1705. [CrossRef]

5. Borowiec, A.; Haugen, H.K. Subwavelength ripple formation on the surfaces of compound semiconductors irradiated with femtosecond laser pulses. Appl. Phys. Lett. 2003, 82, 4462-4464. [CrossRef]

6. Daminelli, G.; Krüger, J.; Kautek, W. Femtosecond laser interaction with silicon under water confinement. Thin Solid Films 2004, 467, 334-341. [CrossRef]

7. Bonse, J.; Krüger, J. Femtosecond laser-induced periodic surface structures. J. Appl. Phys. 2012, 24, 042006-1-042006-7. [CrossRef]

8. Miyazaki, K.; Miyaji, G. Mechanism and control of periodic surface nanostructure formation with femtosecond laser pulses. Appl. Phys. A Mater. Sci. Process. 2014, 114, 177-185. [CrossRef]

9. Miyazaki, K.; Miyaji, G.; Inoue, T. Nanograting formation on metals in air with interfering femtosecond laser pulses. Appl. Phys. Lett. 2015, 107, 071103. [CrossRef]

10. Miyaji, G.; Miyazaki, K. Fabrication of 50-nm period gratings on GaN in air through plasmonic near-field ablation induced by ultraviolet femtosecond laser pulses. Opt. Express 2016, 24, 4648-4653. [CrossRef] [PubMed]

11. Yasumaru, N.; Miyazaki, K.; Kiuchi, J. Glassy carbon layer formed in diamond-like carbon films with femtosecond laser pulses. Appl. Phys. A Mater. Sci. Process. 2004, 79, 425-427. [CrossRef]

12. Miyazaki, K.; Maekawa, N.; Kobayashi, W.; Kaku, M.; Yasumaru, N.; Kiuchi, J. Reflectivity in femtosecond-laser-induced structural changes of diamond-like carbon film. Appl. Phys. A Mater. Sci. Process. 2005, 80, 17-21. [CrossRef]

13. Miyaji, G.; Miyazaki, K. Ultrafast dynamics of periodic nanostructure formation on diamond-like carbon films irradiated with femtosecond laser pulses. Appl. Phys. Lett. 2006, 89, 191902. [CrossRef]

14. Miyaji, G.; Miyazaki, K. Nanoscale ablation on patterned diamond-like carbon film with femtosecond laser pulses. Appl. Phys. Lett. 2007, 91, 123102. [CrossRef]

15. Miyaji, G.; Miyazaki, K. Origin of periodicity in nanostructuring on thin film surfaces ablated with femtosecond laser pulses. Opt. Express 2008, 16, 16265-16271. [CrossRef] [PubMed]

16. Miyaji, G.; Miyazaki, K.; Zhang, K.; Yoshifuji, T.; Fujita, J. Mechanism of femtosecond-laser-induced periodic nanostructure formation on crystalline silicon surface immersed in water. Opt. Express 2012, 20, 14848-14856. [CrossRef] [PubMed]

17. Miyazaki, K.; Miyaji, G. Nanograting formation through surface plasmon fields induced by femtosecond laser pulses. J. Appl. Phys. 2013, 114, 153108. [CrossRef] 
18. Miyaji, G.; Miyazaki, K. Shaping of nanostructured surface in femtosecond laser ablation of thin films. Appl. Phys. A Mater. Sci. Process. 2010, 98, 927-930. [CrossRef]

19. Kärtner, F.X.; Morgner, U.; Schibli, T.; Ell, R.; Haus, H.A.; Fujimoto, J.G.; Ippen, E.P. Few-Cycle Pulses Directly from a Laser. In Few-Cycle Laser Pulse Generation and Its Applications, 1st ed.; Kärtner, F.X., Ed.; Springer: Berlin/Heidelberg, Germany, 2004; pp. 73-136. ISBN 978-3-540-20115-1.

20. Raether, H. Surface Plasmons on Smooth and Rough Surfaces and on Gratings; Springer-Verlag: Heidelberg, Germany, 1988; ISBN 978-3-540-47441-8.

21. Yoshikawa, M.; Katagiri, G.; Ishida, H.; Ishitani, A. Raman spectra of diamondlike amorphous carbon films. J. Appl. Phys. 1988, 64, 6464-6468. [CrossRef]

22. Zhang, S.; Zeng, X.T.; Xie, H.; Hing, P. A phenomenological approach for the Id/Ig ratio and sp3 fraction of magnetron sputtered a-C films. Surf. Coat. Technol. 2000, 123, 256-260. [CrossRef]

23. Robertson, J. Diamond-like amorphous carbon. Mater. Sci. Eng. 2002, R37, 129-281. [CrossRef]

24. Tai, F.C.; Lee, S.C.; Wei, C.H.; Tyan, S.L. Correlation between $I_{\mathrm{D}} / I_{\mathrm{G}}$ Ratio from Visible Raman Spectra and $s p^{2} / s p^{3}$ Ratio from XPS Spectra of Annealed Hydrogenated DLC Film. Mater. Trans. 2006, 47, 1847-1852. [CrossRef]

25. Tuinstra, F.; Koenig, J.L. Raman Spectrum of Graphite. J. Chem. Phys. 1970, 53, 1126-1130. [CrossRef]

26. Nemanich, R.J.; Solin, S.A. First- and second-order Raman scattering from finite-size crystals of graphite. Phys. Rev. B 1979, 20, 392-400. [CrossRef]

27. Yoshikawa, M.; Nagai, N.; Matsuki, M.; Fukuda, H.; Katagiri, G.; Ishida, H.; Ishitani, A.; Nagai, I. Raman scattering from $s p^{2}$ carbon clusters. Phys. Rev. B 1992, 46, 7169-7174. [CrossRef]

28. Ferrari, A.C.; Robertson, J. Interpretation of Raman spectra of disordered and amorphous carbon. Phys. Rev. B 2000, 61, 14095-14107. [CrossRef]

29. Wojdyr, M. Fityk: A general-purpose peak fitting program. J. Appl. Crystal. 2010, 43, 1126-1128. [CrossRef]

30. Miyaji, G.; Miyazaki, K. Role of multiple shots of femtosecond laser pulses in periodic surface nanoablation. Appl. Phys. Lett. 2013, 103, 071910. [CrossRef]

31. Sokolowski-Tinten, K.; Linde, D. Generation of dense electron-hole plasmas in silicon. Phys. Rev. B 2000, 61, 2643-2650. [CrossRef]

32. Because the Drude damping time of the DLC films or GC was never measured, we used the value of Si reported in Ref. 31 . Using the time in a range of 1-10 fs, we confirmed that the calculation results were almost the same as that with the time of $1 \mathrm{fs}$.

33. Alterovitz, S.A.; Savvides, N.; Smith, F.W.; Woollam, J.A. Amorphous Hydrogenated "Diamondlike" Carbon Films and Arc-Evaporated Carbon Films. In Handbook of Optical Constants of Solids; Palik, E.D., Ed.; Academic Press: San Diego, CA, USA, 1985; pp. 838-852. ISBN 978-0-125-44423-1.

34. Rebollar, E.; Pérez, S.; Hernández, J.J.; Martín-Fabiani, I.; Rueda, D.R.; Ezquerra, T.A.; Castillejo, M. Assessment and Formation Mechanism of Laser-Induced Periodic Surface Structures on Polymer Spin-Coated Films in Real and Reciprocal Space. Langmuir 2011, 27, 5596-5606. [CrossRef] [PubMed]

35. Rebollar, E.; Rueda, D.R.; Martín-Fabiani, I.; Rodríguez-Rodríguez, Á.; García-Gutiérrez, M.-C.; Portale, G.; Castillejo, M.; Ezquerra, T.A. In Situ Monitoring of Laser-Induced Periodic Surface Structures Formation on Polymer Films by Grazing Incidence Small-Angle X-ray Scattering. Langmuir 2015, 31, 3973-3981. [CrossRef] [PubMed]

36. Roth, S.V.; Döhrmann, R.; Gehrke, R.; Röhlsberger, R.; Schlage, K.; Metwalli, E.; Körstgens, V.; Burghammer, M.; Riekel, C.; David, C.; et al. Mapping the morphological changes of deposited gold nanoparticles across an imprinted groove. J. Appl. Crystallogr. 2015, 48, 1827-1833. [CrossRef]

(C) 2018 by the authors. Licensee MDPI, Basel, Switzerland. This article is an open access article distributed under the terms and conditions of the Creative Commons Attribution (CC BY) license (http:// creativecommons.org/licenses/by/4.0/). 